\title{
All must have prizes
}

\author{
Millions of dollars of prize money are up for grabs in fundamental physics, through an entrepreneur- \\ funded scheme that should complement, rather than challenge, the Nobel awards.
}

Theoretical physics is unlikely to make you rich - unless you're the lucky winner of one of the recently created Fundamental Physics Prizes ${ }^{1}$. Former physicist turned billionaire investor Yuri Milner hit the headlines last summer when he awarded US $\$ 27$ million to nine physicists for their work on fundamental theory, ranging from particle physics and cosmology to topological quantum computation.

Milner has since established three more prizes: the US $\$ 300,000$ Physics Frontiers Prize; the US\$100,000 New Horizons in Physics Prize; and the Special Fundamental Physics Prize, worth US\$3 million. This last went to Stephen Hawking for his discovery of Hawking radiation and contributions to the investigation of quantum gravity, and to seven leading physicists working with the Large Hadron Collider, for their role "in the scientific endeavour that led to the discovery of the new Higgs-like particle" (which, in light of the most recent data from the CERN experiments, we are now allowed to call "Higgs" rather than "Higgs-like".)

At US\$3 million, the Fundamental Physics Prize far exceeds the cash value of any other prize in physics - including the Nobel Prize, which in 2012 was worth 8 million Swedish Krona, or about US\$1.2 million. The Milner prizes are to be given annually "for accomplishments in fundamental physics broadly defined, including advances in closely related fields with deep connections to physics". Milner and Nobel laureate Steven Weinberg decided the first winners; those chosen then became the selection committee for this year's prize, announced ${ }^{1}$ in late March.

After causing quite a stir with his physics awards, Milner made the news again in February this year, when - with fellow Silicon Valley entrepreneurs, including Mark Zuckerberg of Facebook and Sergey Brin of Google - he announced the names of eleven scientists who would receive US\$3 million each as winners of the new Breakthrough Prize in Life Sciences ${ }^{2}$. The guidelines, and nomination and selection methods for these biology prizes match those for Milner's physics awards.

Reactions to the inaugural prizes in both physics and the life sciences have ranged from sharp criticism to highly enthusiastic praise, and even the dubbing of Milner's scheme as the "Nobels 2.0". The terms of the Nobel awards, however, were firmly laid out in the will of Alfred Nobel: the long-term future of the new awards will depend on the inclination - and good fortune - of their patrons, and only time will tell whether they can indeed come to rival the prestige of the Nobel prizes. But more interesting than the publicity and the sums of money involved is the way these awards challenge the tradition in science of how breakthroughs are recognized.

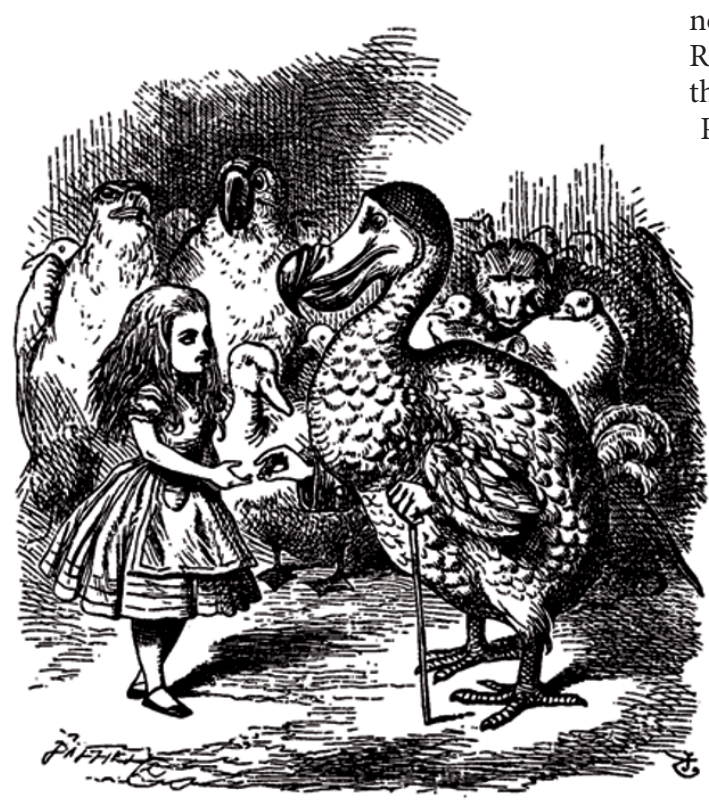

Nobel prizes may not be divided between more than three people ${ }^{3}$ - and yet, in the century since their launch, science has become an increasingly collaborative endeavour. Of course, there are still many achievements in physics that are recognizably attributable to the efforts or foundation-laying work of particular individuals. But, across all of science, there are breakthroughs that rest on the collaborative success of more than three individuals. In contrast to the Nobels, any number of people may share a Fundamental Physics Prize, and Milner and colleagues have already grasped the CERN 'thorn' by identifying six leaders of the experimental collaborations alongside the man who led the LHC construction project, Lyn Evans, as winners for work that has made a Higgs discovery possible. One imagines, too, that this prizegiving committee, unlike the Nobel one, may avoid the similarly thorny issue of which of six theorists involved in the origination of the Higgs mechanism might deserve reward.

And although the Nobel Committee casts its net wide in seeking candidates for their prize, the Milner net is wider. About 3,000 scientists take part in a five-month-long international process to produce the Nobel nominations $s^{4}$, from which ultimately the Royal Swedish Academy of Sciences choses the laureates. However, for the Fundamental Physics Prize, anyone may make an online nomination; past recipients then decide the winners.

The new system of awards does indeed raise timely questions about the assessment of scientific merit. Certainly a proliferation akin to that advocated by the Dodo in Lewis Carroll's Alice's Adventures in Wonderland (pictured) is in no one's interests: adjudicating a race in which competitors ran in circles, however far and for however long they wished, the Dodo declared, "Everybody has won and all must have prizes."

That would be meaningless, but all should have the possibility of prizes. The most striking feature of Milner's awards in physics is their acknowledgement of the kind of theoretical work - particularly string theory - that struggles to be noticed by the Nobels. (New work led by Milner laureate Nima Arkani-Hamed is discussed elsewhere in this issue ${ }^{5}$.)

The Fundamental Physics Prizes may be bigger but they are not necessarily better than the long-standing Nobels - nor need they be. As science evolves - what we do, how we do it and, yes, how we publish it - it's natural that how we recognise achievement and excellence will evolve too.

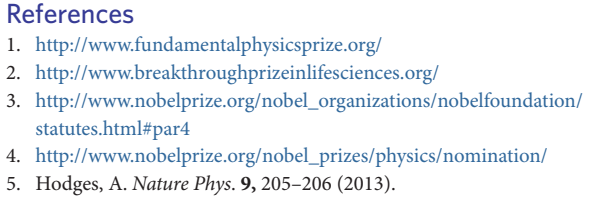

